\title{
PAL APPLICATION TO THE STUDY OF SORPTION MECHANISM IN POLYMERS - CAPILLARY EFFECTS
}

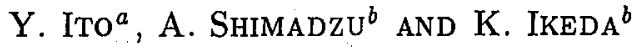 \\ ${ }^{a}$ Research Center for Nuclear Science and Technology \\ The University of Tokyo, Tokai, Ibaraki 319-11, Japan \\ ${ }^{b}$ Nitto Denko Corporation, 1-1-2, Shimohozumi, Ibaraki, Osaka 567-8680, Japan
}

The positron annihilation lifetime technique can be applied to the study of the sorption mechanism in polymers in a quite unique way. In our previous experiments it had been shown that $\tau_{3}$ and $I_{3}$ of polymers show a $\mathrm{V}$-shaped dependence as a function of the contact with vapors. The decreasing part of the V-shaped dependence had been attributed to the Langmuir-type sorption, and the increasing part to delayed occurrence of the Henry-type sorption. But since there was some doubt that a capillary effect, i.e. the vapor to be sorbed is deposited in between the polymer membranes, might be involved in the increasing part, we performed a careful experiment to avoid the capillary effect. We have performed further experiments in a careful condition to avoid the capillary effect, and have observed the same V-shape as before. Thus our interpretation of the V-shaped dependence has been established. Furthermore, in another example it is shown a case where the capillary effect is observed. This latter case is an example how positron annihilation lifetime can distinguish the real sorption and false sorption (capillary effect).

PACS numbers: $78.70 . \mathrm{Bj}$

\section{Introduction}

We demonstrated before [1] that positron annihilation lifetime (PAL) can be used to study the mechanism of vapor sorption in polymers in an unparalleled way. For rubbery polymers the Henry-type sorption overwhelms from the beginning of sorption and both $\tau_{3}$ and $I_{3}$ increase steadily with the contact time. For glassy polymers the Langmuir-type sorption takes place at first causing decreases of $\tau_{3}$ and $I_{3}$, and later they start to rise due to delayed occurrence of Henry-type sorption. Thus the plot of $\tau_{3}$ and $I_{3}$ vs. the contact time with vapors in glassy polymers shows a $V$-shaped dependence. But in fact the behavior described above corresponds to typical cases. Quite frequently $\tau_{3}$ and $I_{3}$ do not change in the same direction during the course of sorption. For example, by the sorption of benzene in polycarbonate (a glassy polymer) $I_{3}$ decreases as expected but $\tau_{3}$ increases 
instead [2]. This was supposed to be due to "local plasticization" of the glassy polymer, but a detailed examination is necessary.

In this report we describe our recent results on this line. In our former experiments we had something to worry about in the experimental technique. We used stacked thin polymer films as the sample to ensure rapid diffusion of the vapor molecules and the vapor was supplied from a liquid reservoir connected to the sample chamber. In this condition it is probable that the vapor molecules deposit as liquid between the polymer sheets (capillary effect) and $\tau_{3}$ and $I_{3}$ rise simply because some fractions of positrons are stopping in the liquid. If this had occurred it could have been misinterpreted for the delayed occurrence of the Henry-type sorption. In order to avoid such possible capillary effects we have performed a careful experiment and have confirmed that the interpretation of the delayed Henry-type sorption is valid. We further report a particular case in which the capillary effect has been observed to be really taking place for polymers having a more fabric structure. In this case PAL serves as an excellent "false detector" of sorption.

\section{Experimental}

The experimental set-up was fundamentally the same as reported before $[1,2]$. Two glass tubes having vacuum stop-cocks were connected together. The positron source-sample sandwich was put in one arm, and liquid was put in another. After evacuating the whole system, the vapor was introduced in the sample. The tube containing the liquid was being placed in a small bath using Peltier effect, the liquid temperature could be maintained below room temperature. The vapor pressure was thus kept lower than the equilibrium pressure, which ensures absence of the possible capillary effect. Polycarbonate was chosen as the sample for this experiment. The sample for which a capillary effect was observed was a kind of cross-linked polyamide film specially manufactured for the purpose of reverse osmosis membrane*. The membrane has a pleat-like skin layer of up to about $0.4 \mu \mathrm{m}$.

\section{Results and discussion}

The results for polycarbonate with benzene as the vapor are shown in Fig. 1a and $b$. The two figures correspond to different series of experiments using different polycarbonate samples. The contact of the vapor with the sample was started at time zero, and the temperature of the liquid was changed at appropriate times as shown in the figure, the lower temperature corresponding to lower vapor pressure: e.g. 35 torr at $6^{\circ} \mathrm{C}, 75$ torr at $20^{\circ} \mathrm{C}$. At each temperature the data point is seen to reach a level-off value after 8-10 hours. This means that it requires such a long time for the sorption to reach an equilibrium state. The $o$-Ps lifetime de- . creased slightly at 6 and $9{ }^{\circ} \mathrm{C}$, and turned to rise above $12^{\circ} \mathrm{C}$. Evidently there is a $\mathrm{V}$-shaped dependence between $\tau_{3}$ and the vapor pressure corresponding to an initial Langmuir-type sorption and delayed Henry-type sorption. The value of $I_{3}$. does not show a V-shaped dependence however.

*ES-series RO-membranes manufactured by Nitto Denko Co. 


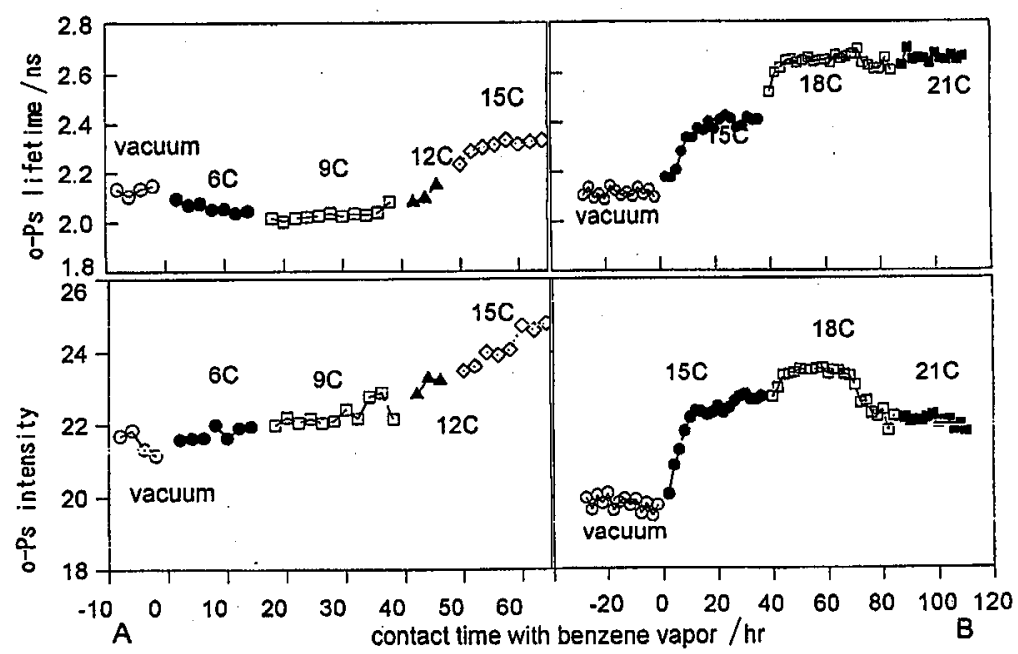

Fig. 1. o-Ps lifetime (upper) and intensity (below) in polycarbonate exposed to benzene vapor which was supplied from a liquid benzene reservoir. The temperature of the liquid reservoir is indicated in the figure, and the room temperature is around $20^{\circ} \mathrm{C}$.

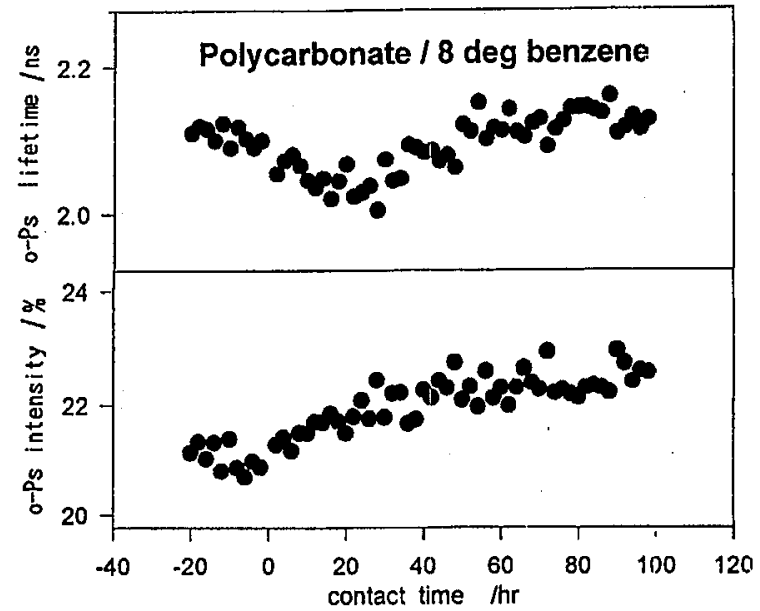

Fig. 2. o-Ps lifetime and intensity in polycarbonate exposed to benzene vapor supplied from $8^{\circ} \mathrm{C}$ benzene liquid.

In our previous paper [2] the $\mathrm{V}$-shape was shown as a function of the contact time. This can also be seen for the polycarbonate/benzene system when the vapor pressure is suitably selected. The result obtained at $8^{\circ} \mathrm{C}$ is shown in Fig. 2. A small $\mathrm{V}$-shape is seen for $\tau_{3}$, but $I_{3}$ only increases slightly. When the vapor pressure was higher, however, $\tau_{3}$ simply increased without showing a $V$-shape dependence; because the sorption proceeded rapidly.

Thus it has been confirmed that the rise in the case of $\tau_{3}$ and $I_{3}$ is not due to the capillary effect. Local plasticization at the site of sorption may still be a possible explanation. 
The occurrence of the capillary effect was found in another series of experiments using engineering polymer materials. A part of the results is shown in Fig. 3. When the sample (ES-10 and ES-20 are the manufacturer's naming for the RO-membrane) was contacted with hexane vapor (Fig. 3a), $\tau_{3}$ increased while $I_{3}$ decreased slightly. When water vapor was used (Fig. $3 \mathrm{~b}$ ), however, no change was occurred for $\tau_{3}$ and $I_{3}$. The sorption was also studied by weight measurement, according to which water appeared to be absorbed quite efficiently (Fig. 4). Apparently the PAL data and the weight data are contradictory, and a plausible solution should be the capillary effect. Water may be depositing among the pleat structure of the polymer skin. If we examine Fig. $3 \mathrm{~b}$ carefully we notice that $I_{3}$ is slightly
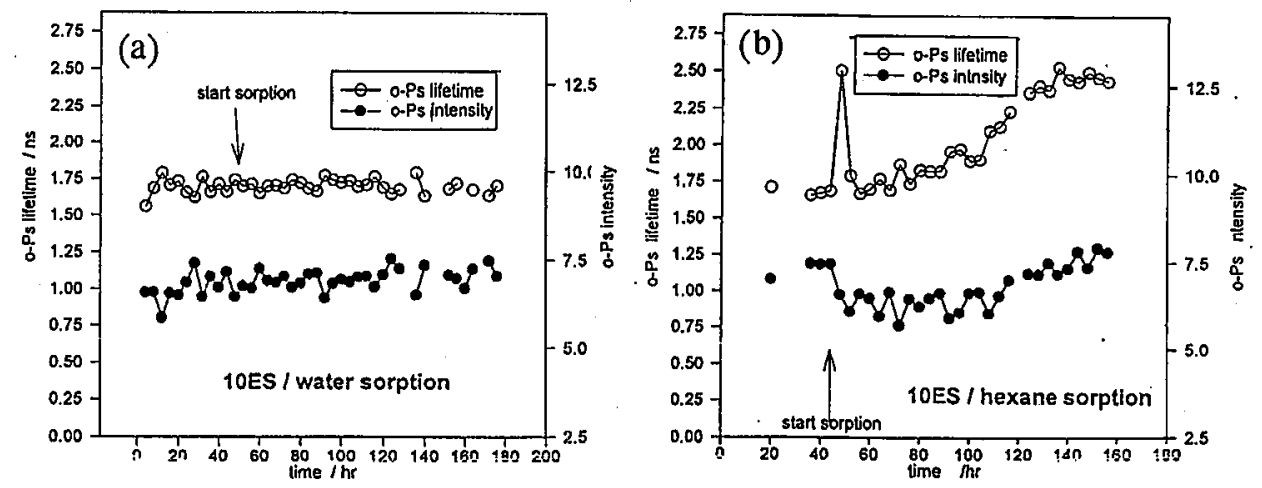

Fig. 3. o-Ps lifetime and intensity in reverse osmosis membrane made from cross-linked polyamide exposed to hexane (b) or water vapor (a) with the pressure equilibrium at room temperature. The data for water indicates that the polymer is not sorbing water.

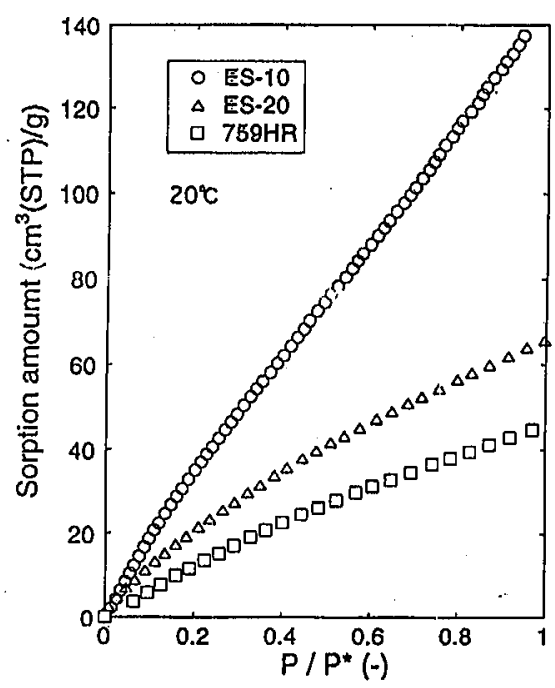

Fig. 4. Water sorption data measured by weight indicating that ES-10, the same polymer as shown in Fig. 3, is sorbing a substantial amount of water. 
increasing. This is probably due to the sorbed water. This is a good and simple example of practical utilization of the PAL technique to the sorption-related problem. Only with Fig. 4 one would be lead to think that water is highly hydrophilic, and with the aid of Fig. $3 \mathrm{~b}$ it is only possible to imagine water depositing on the surface.

In Fig. 3 there is a sharp rise in $\tau_{3}$ at the very beginning of contact with hexane vapor. It is not the purpose of this paper to discuss this, but we just mention that this appears to be real since the same was observed for other experiments using the similar series of polymers. Probably the polymer chains are stressed on contacting with the vapor molecules.

\section{Conclusion}

Since positronium efficiently detects vacancy sites related with sorption, it is a powerful tool of studying sorption. It can distinguish Langmuir- and Henry-type sorption, and also can "false detect" a capillary effect. It is important to perform PAL experiments of sorption ensuring that the capillary effect is not taking place.

\section{References}

[1] Y. Ito, V. Sanchez, R. Lopez, L.A. Fucugauchi, K. Tanaka, K. Okamoto, Bull. Chem. Soc. Jpn. 66, 727 (1993).

[2] Y. Ito, H.F.M. Mohamed, K. Tanaka, K. Okamoto, J. Radioanal. Nucl. Chem. 211, 211 (1996). 